\title{
Ulcère de Buruli : Connaissance et Rôle des Plantes dans la Transmission de la Maladie dans Quatre Villages des Districts Sanitaires de Daloa et Bouaké (Côte d'Ivoire)
}

\author{
Ehouman Evans, (Doctorant) \\ Soro Dramane, (Maître-Assistant)
}

UFR des Sciences Biologiques, Université Peleforo Gon Coulibaly de

Korhogo, BP Korhogo, Côte d'Ivoire

Bakayoko Adama, (Professeur Titulaire)

Koné Mamidou Witabouna, (Professeur Titulaire)

UFR des Sciences de la Nature, Université Nangui Abrogoua, Côte d'Ivoire

Doi:10.19044/esj.2019.v15n21p181 URL:http://dx.doi.org/10.19044/esj.2019.v15n21p181

Résumé

L'ulcère de Buruli est une maladie tropicale négligée qui se manifeste par des ulcérations graves de la peau, des muscles et des os, pouvant conduire à des amputations. Des efforts ont été faits pour éradiquer cette maladie. Cependant, de nouveaux cas sont diagnostiqués chaque année et les foyers endémiques ne font que se multiplier. La pénétration dans l'organisme de l'agent pathogène, Mycobacterium ulcerans, se ferait suite à une effraction cutanée causée notamment par les plantes. L'objectif de ce travail est d'évaluer le niveau de connaissance de l'ulcère de Buruli des populations et du rôle des plantes dans la transmission de cette maladie afin de mieux sensibiliser sur les modes de prévention. Des entretiens basés sur la méthode Connaissance, Attitudes et Pratiques (CAP) ont été réalisés dans les Districts sanitaires de Daloa et de Bouaké (Côte d'Ivoire). Les données démographiques, la connaissance des signes, des causes et des appellations de l'ulcère de Buruli dans les langues locales ont été obtenues. Il en ressort que les populations ont une connaissance fragmentaire de l'ulcère de Buruli. Les appellations de la maladie sont liées à la taille de la plaie sur la peau. Les populations ont identifié des espèces végétales telles que Elaeis guineensis, Imperata cylindrica, Chromolaena odorata et Combretum racemosum comme pouvant causer des effractions cutanées. La cause mystique de la maladie est fréquente. Il serait donc urgent d'accentuer les campagnes de sensibilisation dans les zones endémiques d'ulcère de Buruli. 
Mots-clés : Côte d'Ivoire, Ulcère de Buruli, Mycobacterium ulcerans, plantes, transmission

\title{
Knowledge of Buruli Ulcer and Role of Plants in Disease Transmission in Four Villages Belonging to Health Districts of Daloa and Bouaké (Côte d'Ivoire)
}

\author{
Ehouman Evans, (Doctorant) \\ Soro Dramane, (Maître-Assistant) \\ UFR des Sciences Biologiques, Université Peleforo Gon Coulibaly de \\ Korhogo, BP Korhogo, Côte d'Ivoire \\ Bakayoko Adama, (Professeur Titulaire) \\ Koné Mamidou Witabouna, (Professeur Titulaire) \\ UFR des Sciences de la Nature, Université Nangui Abrogoua, Côte d'Ivoire
}

\begin{abstract}
Buruli ulcer (BU) is a neglected tropical disease leading to severe ulcerations of the skin, muscles and bones leading to amputations if not earlier detected. Despite efforts to eradicate this disease, new cases are diagnosed every year and endemic foci are increasing. The penetration into the body of the pathogen agent Mycobacterium ulcerans, would occur after a skin injures due to plants materials. The objective of this work was to assess the level of knowledge of the populations and the role that plants could play in the transmission of Buruli ulcer. Interviews were conducted in four villages belonging to two Health Districts in Côte d'Ivoire, based on knowledge, attitude, and the practice (KAP) method. Demographic data set, knowledge of the signs, causes and names of Buruli ulcer in the local language were obtained. Plants that can cause injuries have been identified. It appears that populations have a fragmentary knowledge of Buruli ulcer. The names of the disease are related to the size of the wound on the skin. Populations have identified plant species such as Elaeis guineensis, Imperata cylindrica, Chromolaena odorata and Combretum racemosum as causing openings on the skin. The mystical cause of the disease is frequent. Thus, it would therefore be urgent to increase awareness campaigns in the endemic areas of Buruli ulcer.
\end{abstract}

Keywords: Côte d'Ivoire, Buruli ulcer, Mycobacterium ulcerans, Plants, Transmission 


\section{Introduction}

L'ulcère de Buruli est une maladie tropicale nécrotique dont l'agent pathogène est une mycobactérie environnementale, appelée Mycobacterium ulcerans (MU). Elle est la troisième maladie à Mycobacterium après la tuberculose et la lèpre, avec une forte occurrence dans les zones humides, en régions endémiques (Hotez \& Kamath, 2009 ; Sopoh \& Asiedu, 2016).

Cette maladie constitue un problème de santé publique dans près de 30 pays du monde, situés dans la zone intertropicale, aussi bien en Afrique, en Amérique, en Asie qu'en Océanie. L'Afrique est le continent où on note le plus grand nombre de cas. Bien que le nombre de nouveaux malades tende à diminuer dans certains pays, on observe l'apparition de la maladie dans des pays qui jusqu'alors n'avaient enregistré aucun cas. (WHO, 2017).

Dans les pays africains en développement où sévit l'ulcère de Buruli, les personnes affectées par la maladie sont victimes d'invalidité, de réduction de l'employabilité, d'interruption scolaire, de moquerie, de divorce ou d'abandon des membres de leur famille et de leur communauté (Tschakert et al., 2016). Pour la plupart des communautés villageoises, cette maladie serait liée aux pratiques magico-occultes. Il en résulte une marginalisation d'une partie de la population que l'on qualifie de sorcière, accusée de transmettre la maladie aux autres (Giles-Vernick et al., 2015 ; Koka et al., 2016).

En Côte d'Ivoire, 24000 cas ont été enregistrés entre 1978 et 2006. Aussi, plus de 9000 cas ont été enregistrés uniquement pour la période de 2007 à 2010, avec plus de 2600 cas en 2009. À ce jour, la Côte d'Ivoire demeure le pays le plus affecté avec 376 nouveaux cas par an, suivi du Ghana et du Bénin (WHO, 2017).

En dépit des nombreuses études qui ont été entreprises pour pallier cette crise sanitaire et des progrès réalisés ces dernières années qui ont permis d'isoler et de caractériser M. ulcerans à partir de sources environnementales (Ross et al., 1997 ; Portaels et al., 2008 ; Tai et al., 2018), force est de constater que le mode de transmission de la maladie n'est toujours pas clairement élucidé et de nouveaux foyers endémiques se développent (Garchitorena et al., 2015 ; Khanna et al., 2016). Toutefois, il est clairement admis que l'infection interviendrait par contact direct avec le réservoir environnemental à la faveur soit de coupures ou de blessures soit de piqûre d'insectes aquatiques (Merritt et al., 2010 ; Williamson et al., 2014 ; O’Brien et al., 2019). Dans cette transmission, les plantes se sont révélées être porteuses de $M$. ulcerans (McIntosh et al., 2014 ; Boni et al., 2017). Aussi, les blessures liées aux plantes se sont révélées des facteurs à risque (Zogo et al., 2015). La transmission d'homme à homme semblerait rare (Röltgen \& Pluschke, 2015). En Côte d'Ivoire, les travaux de Tano et al. (2017) ont mis en évidence, la présence de $M$. ulcerans sur des biofilms de plantes. 
Les Districts sanitaires de Bouaké et de Daloa constituent les pôles de plus forte endémicité en Côte d'Ivoire. Historiquement, c'est à Daloa qu'ont été enregistrés les flambées de cas d'ulcère de Buruli (Marston et al., 1995). À ce jour, les Districts sanitaires de Daloa et de Bouaké abritent deux Centres de références en matière de traitement de l'ulcère de Buruli, à savoir le Centre Jean-Baptiste Vatelot à Bouaké et le Centre Saint Michel dans la commune de Zoukougbeu.

À ce jour, aucune étude ne s'est intéressée au rôle que pourraient jouer les plantes dans la transmission de la maladie. Les retombées de cette étude devraient permettre de connaître l'état actuel du niveau de connaissance de l'ulcère de Buruli dans les zones endémiques et de pouvoir orienter les campagnes de sensibilisation auprès de toutes les couches de la population.

L'objectif de ce travail est d'évaluer le niveau de connaissance des populations sur le mode de contamination et le rôle des plantes dans la transmission de l'ulcère de Buruli, en vue de mettre fin à la chaîne de transmission de cette maladie. L'étude a été menée dans les zones hyperendémiques et les zones hypoendémiques d'ulcère de Buruli choisis sur la base des informations obtenues par les points focaux dans les différents Districts sanitaires.

\section{Matériel et méthodes \\ Sites d'étude et échantillonnage}

Cette étude a été menée dans les villages de Kongodékro et Konankro dans le District sanitaire de Bouaké et dans les villages de Batéguédia et Zébra dans le District sanitaire de Daloa (figure 1). Ces Districts ont été choisis compte tenu de leur prévalence élevée pour l'ulcère de Buruli. En effet, pour une population totale de 591633 et 680694 habitants respectivement pour les départements de Daloa et Bouaké (INS, 2014), 225 et 130 cas d'ulcère de Buruli ont été enregistrés, ce qui correspond à une prévalence de 38,03 pour 100000 habitants dans le District sanitaire de Daloa et 19,09 pour 100000 habitants dans les Districts sanitaires de Bouaké Nord Est, Nord-Ouest et Sud (PNLUB, 2013 ; 2014).

Dans cette étude, les caractères hyperendémique et hypoendémique sont définis suite à un entretien semi-directif avec les points focaux dans les différents Districts sanitaires. Ils correspondent respectivement, aux villages pour lesquels plus de cinq cas d'ulcère de Buruli ont été enregistrés et ceux pour lesquels moins de cinq cas ont été notifiés.

\section{Enquêtes}

Les enquêtes sur les connaissances, attitudes et pratiques (CAP), ont été menées auprès des populations dans les sites hyperendémiques (villages de Batéguédia et Konankro) et ceux hypoendémiques (Zébra et Kongodékro). 


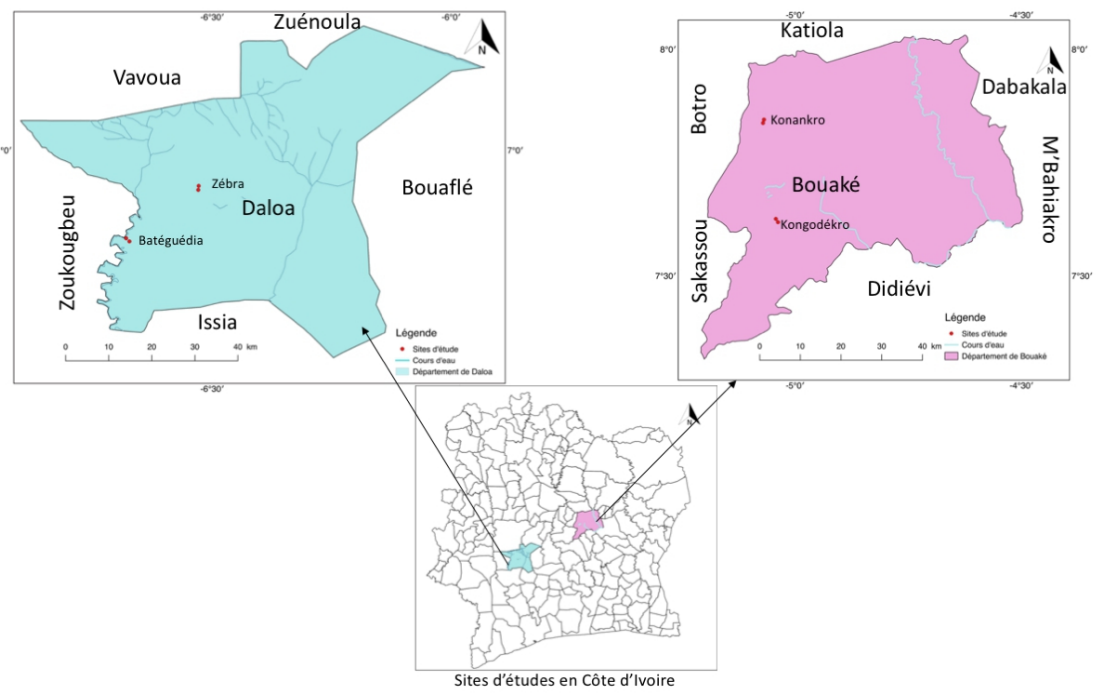

Figure 1 : Situation géographique des départements de Bouaké et de Daloa

Il s'agit d'une enquête mixte qui a consisté en des entretiens directifs ou structurés, à partir d'un questionnaire et des entretiens semi-structurés à l'aide d'un guide (De Ketele \& Roegiers, 2015 ; De Zeeuw et al., 2015 ; Garapati et al., 2018). Cette approche est inspirée de celle employée lors des études CAP de Akoachere et al. (2016) au Cameroun et par Handicap (2013) au Togo dans le cas de l'étude de l'ulcère de Buruli ainsi que de Adjet (2017) en Côte d'Ivoire. Cette approche mixte présente l'avantage de pouvoir soumettre les réponses à une analyse statistique inférentielle (Yusra et al., 2014).

Les entretiens ont parfois été réalisés avec l'aide d'un interprète, lorsque la personne enquêtée ne s'exprimait pas en français. Le questionnaire a comporté trois parties principales : les données démographiques (sexe, âge et profession), les données relatives à la connaissance de l'ulcère de Buruli (existence de cas dans le village, signes de l'ulcère de Buruli, mode de transmission de l'ulcère de Buruli) et les données relatives au rapport entre l'ulcère de Buruli et les plantes (connaissance de la flore de la région, existence de lien entre les plantes, les blessures et l'ulcère de Buruli). Les entretiens semi-structurés ont porté sur la nomenclature de l'ulcère de Buruli et la connaissance des plantes que les populations estiment à risque dans la transmission de la maladie. Pour calculer la taille de l'échantillon, le logiciel statistique Epi Info a été utilisé pour une population globale de 7000 habitants (INS, 2015a ; 2015b) avec une marge d'erreur de $10 \%$. Ainsi, une taille d'échantillon minimale de 115 personnes avec un intervalle de confiance de $97 \%$ a été obtenue. Ces personnes ont été choisies de manière aléatoire. Les critères d'inclusion retenus sont les suivants : les sujets des deux sexes, être 
âgé de 10 ans au moins, pouvoir s'exprimer verbalement, souffrir ou non de l'ulcère de Buruli et avoir donné son consentement préalable. Les personnes ne remplissant pas ces conditions ont été exclues de l'étude.

\section{Analyse des données}

Le logiciel Epi Info ${ }^{\mathrm{TM}}$, version 7.2.0.1 (CDC ; Center for Disease Control, Atlanta, United State of America) a été utilisé pour la constitution du formulaire pour l'interview, pour l'enregistrement et l'analyse des données. Le logiciel Excel de Microsoft a été utilisé pour la réalisation des graphiques. Afin de mettre en relation entre les réponses des personnes enquêtées et le statut du site, un test exact de Fisher a été effectué avec un seuil de significativité de $5 \%$ (Cooper et al., 2017).

\section{Résultats}

\section{Caractéristiques démographiques des populations enquêtées}

Dans le tableau I sont consignées les caractéristiques sociodémographiques des personnes enquêtées. Un total 121 personnes, provenant de quatre villages a été interrogé, en moyenne $66,94 \%$ sont des hommes et $33,06 \%$ sont des femmes. La plupart des personnes enquêtées pratiquent l'agriculture $(59,54 \%)$. La tranche d'âge la plus importante des personnes interrogées est celle se situant entre 25 et 36 ans $(37,29 \%)$. Les résultats montrent qu'en moyenne $13,30 \%$ des personnes interrogées ont moins de 16 ans. Il existe une différence significative entre la proportion de personnes affectées par l'ulcère Buruli et les personnes non affectées selon les différentes tranches d'âge $(P<0,001)$ (Tableau II).

Tableau I : Caractérisation sociodémographiques des populations enquêtées

\begin{tabular}{|c|c|c|c|c|c|}
\hline \multirow[b]{2}{*}{ Paramètre } & \multicolumn{2}{|c|}{ Faible endémicité } & \multicolumn{2}{|c|}{ Forte endémicité } & \multirow[b]{2}{*}{ Moyenne } \\
\hline & Zébra & Kongodékro & Batéguédia & Konankro & \\
\hline Effectif & 31 & 30 & 30 & 30 & \\
\hline Genre (\%) & & & & & \\
\hline Femme & 32,26 & 33,33 & 36,67 & 30 & 33,06 \\
\hline $\begin{array}{l}\text { Homme } \\
\text { Tranches d'âge en années } \\
(\%)\end{array}$ & 67,74 & 66,67 & 63,33 & 70 & 66,94 \\
\hline Moins de 16 & 3,23 & 3,33 & & 33,33 & 13,3 \\
\hline $16-25$ & & & 3,33 & & 3,33 \\
\hline $25-36$ & 25,81 & 60 & 36,67 & 26,67 & 37,29 \\
\hline $36-45$ & 45,16 & 23,33 & 36,67 & 10 & 28,79 \\
\hline 45 et plus & 25,81 & 13,33 & 23,33 & 30 & 23,12 \\
\hline
\end{tabular}

Activité Professionnelle (\%) 
Agent de Santé

Élève

Fonctionnaire

Instituteur

Ménagère

Planteur

Retraité

63,33

UB + : malade de l'ulcère de Buruli ; UB- : non malade de l'ulcère de Buruli

Tableau II : Proportion de malade d'ulcère de Buruli en fonction de l'âge

\begin{tabular}{lccc}
\hline Tranches d'âge (années) & UB+ n (\%) & UB- n $(\%)$ & P-value \\
\hline Moins de 16 & $10(37,04)$ & $2(2,13)$ & $<0,001$ \\
$16-25$ & $0(00)$ & $1(1,06)$ & \\
$25-36$ & $8(29,63)$ & $37(39,36)$ & \\
$36-45$ & $6(22,22)$ & $29(30,85)$ & \\
45 et plus & $3(11,11)$ & $25(26,6)$ & \\
\hline Total & $27(100)$ & $94(100)$ \\
\hline \multicolumn{4}{c}{ UB + : malade de l'ulcère de Buruli ; UB- : non malade de l'ulcère de Buruli } \\
\multicolumn{4}{c}{ n : effectif }
\end{tabular}

\section{Appellation de l'ulcère de Buruli}

Le tableau III présente les appellations de l'ulcère de Buruli dans quelques langues locales de la Côte d'Ivoire. Dans cette étude, $100 \%$ des personnes interrogées connaissent l'appellation de l'ulcère de Buruli soit dans leur langue locale, soit dans la langue de la région. Chez les Baoulé du sousgroupe Fahafouè dans la région de Bouaké, par exemple, l'ulcère de Buruli est connu sous trois appellations. La première appellation est « Daloa Kani », ce qui se traduit en français par plaie de Daloa, car selon les personnes interrogées, «c'est une plaie qui vient de Daloa, c'est là-bas qu'on a découvert ça ». La seconde appellation est «Kanitè », ce qui se traduit par mauvaise plaie. En effet, pour les populations, «la plaie a une mauvaise apparence, elle est vilaine ", la maladie «fait souffrir la personne affectée, elle est préoccupée, inquiète ». L'ulcère de Buruli porte aussi le nom de «Kanidan», ce qui veut dire grosse plaie, en effet, «tu vois que la plaie grossit et affecte tout le membre ou n'importe quelle partie du corps, le pied, la main et même le ventre ou le dos ». À Daloa, il existe plusieurs appellations pour l'ulcère de Buruli, du fait de la diversité des groupes ethniques des personnes interrogées. Chez les Bété, les Malinké, les Niaboua et les Senoufo, les appellations signifient "grosse plaie ». Il s'agit respectivement de Gbiakada, Djoliba, Djiakabga et Nomkpok. 
Tableau III : Noms vernaculaires de l'ulcère de Buruli dans les localités de Bouaké et de Daloa en Côte d'Ivoire

\begin{tabular}{lll}
\hline Localités d'enquête & Langues & Appellation \\
\hline Bouaké & Baoulé & Daloa kani, Kani dan, Kani tè \\
& Bété & Gbiakada, Vaguegbe \\
& Niaboua & Djiakagba \\
Daloa & Gouro & Mondrelè \\
& Malinké & Djoliba \\
& Senoufo & Namkpok \\
\hline
\end{tabular}

\section{Connaissance de l'ulcère de Buruli}

Pour l'ensemble des sites d'étude, seulement $0,83 \%$ ne connaissent pas l'existence de l'ulcère de Buruli, contre $99,17 \%$ des personnes interrogées qui ont eu une expérience de la maladie, soit par eux-mêmes, soit par le biais des personnes de leur entourage à savoir amis, parents ou connaissances quelconques du voisinage.

Sur l'ensemble des personnes interrogées dans les zones de forte endémicité à savoir les villages de Konankro (Bouaké) et de Batéguédia (Daloa), 98,10\% des répondants connaissent l'ulcère de Buruli. Dans les zones de faible endémicité que sont les villages de Kongodékro (Bouaké) et Zébra (Daloa), à la question de savoir s'ils connaissent la maladie, $100 \%$ des personnes affirment connaitre l'ulcère de Buruli : "Oui nous avons entendu parlé, soit à la radio. La fille du voisin a eu cette maladie » (figure 2 ).

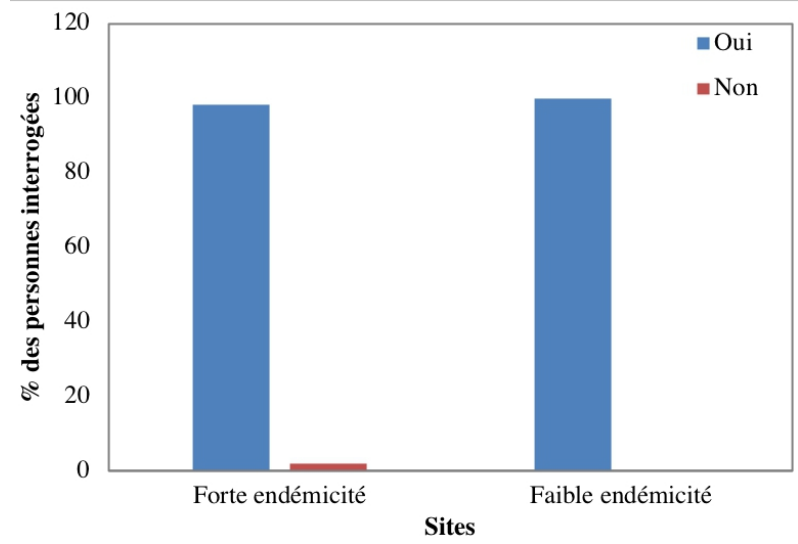

Figure 2 : Répartition des personnes connaissant l'ulcère de Buruli dans les sites de forte et de faible endémicité

\section{Relation ulcère de Buruli et plantes}

Dans les zones où les enquêtes ont été réalisées, 91,74\% des personnes interrogées savent différencier les plantes qu'elles rencontrent dans 
leur milieu. Huit (8) espèces végétales ont été incriminées par les populations, comme susceptibles de causer des coupures. Il s'agit de Elaeis guineensis Jacq. (Arecaceae), Imperata cylindrica (L.) Raeusch. (Poaceae), Chromolaena odorata (L.) R. M. King \& H. Rob. (Asteraceae), Combretum racemosum P. Beauv. (Combretaceae), Ceiba pentandra (L.) Gaertn. (Malvaceae), Dioscorea sp. L. (Dioscoreaceae), Saccharum officinarum L. (Poaceae) et Scleria depressa (C. B. Clarke) Nelmes (Cyperaceae). En effet, 33,33 \% des personnes souffrant de l'ulcère de Buruli dans cette étude affirment que les premiers signes de la maladie sont apparus après qu'elles aient été blessées par l'une de ces plantes lors des travaux champêtres. La liste de ces espèces végétales avec les noms en langue vernaculaire ou communs figurent dans le tableau IV.

$\underline{\text { Tableau IV : Nom de quelques plantes citées par les populations causant des blessures }}$

\begin{tabular}{lll}
\hline Espèces végétales & Langue & $\begin{array}{l}\text { Nom vernaculaire ou } \\
\text { commun }\end{array}$ \\
\hline $\begin{array}{l}\text { Chromolaena odorata } \text { (L.) R. M. } \\
\text { King \& H. Rob. }\end{array}$ & Baoulé & Sékou Touré / indépendance \\
$\begin{array}{l}\text { Elaeis guineensis Jacq. } \\
\text { Combretum racemosum P. Beauv. }\end{array}$ & Baoulé & N'mé \\
$\begin{array}{l}\text { Imperata cylindrica } \text { (L.) P. Beauv. } \\
\text { Saccharum officinarum } \text { L. }\end{array}$ & Baoulé & Flon \\
Ceiba pentandra (L.) Gaertn. & Baoulé & Canne à Sucre/Anglannanh \\
$\begin{array}{l}\text { Combretum racemosum } \text { P. Beauv. } \\
\text { Dioscorea } \text { sp. L. }\end{array}$ & Bété & N'gninh \\
\hline
\end{tabular}

\section{Connaissance des manifestations de la maladie}

Les données relatives à la connaissance des manifestations de l'ulcère de Buruli dans les localités de faible et de forte endémicité sont indiquées à la figure 3 .

Dans les localités de forte endémicité, les personnes interrogées ont été en mesure d'identifier des manifestations de la maladie. Les enflures (68 $\%)$, l'œdème $(60,00 \%)$, le nodule $(60,00 \%)$ et l'ulcération $(56,67 \%)$ constituent les symptômes les plus cités dans les sites de forte endémicité. Dans les localités de faible endémicité, ce sont les enflures (49,18\%), les nodules $(36,7$ \%) et les douleurs $(36,07$ \%). Il existe des symptômes qui n'ont pas été identifiés dans la littérature comme la diarrhée et les vomissements que les populations ont cités lors de cette étude, respectivement par 4,92\% et 1,64 $\%$ des enquêtés dans les sites de faible endémicité ainsi que par 1,67\% pour la diarrhée dans les sites de forte endémicité. 


\section{Causes de la maladie}

La cause mystique de la maladie est la plus récurrente pour 24,43\% des personnes interrogées. Le rôle des sortilèges comme agent causal dans la transmission de la maladie est différemment incriminé selon l'endémicité forte ou faible du village visité $(P=0,0009)$. Ainsi, pour $68,85 \%$ des personnes dans les sites de faible endémicité, l'ulcère de Buruli est causé par des sortilèges contre 36,67 \% dans les sites de forte endémicité. Pour 33,88 \% des personnes enquêtées, cette maladie a une cause végétale, c'est-à-dire elle peut être causée par une plante et $4,96 \%$ une origine alimentaire. Dans les sites de forte endémicité, ce sont $23,33 \%$ des personnes interrogées qui n’ont pas de connaissance sur des causes de l'ulcère de Buruli. Ce pourcentage est plus faible dans les sites de faible endémicité, où il est de 6,56\%. Il existe une différence significative entre la proportion des personnes connaissant l'ulcère de Buruli entre les villages de forte endémicité et les villages de fiable endémicité $(P=0,01)$.

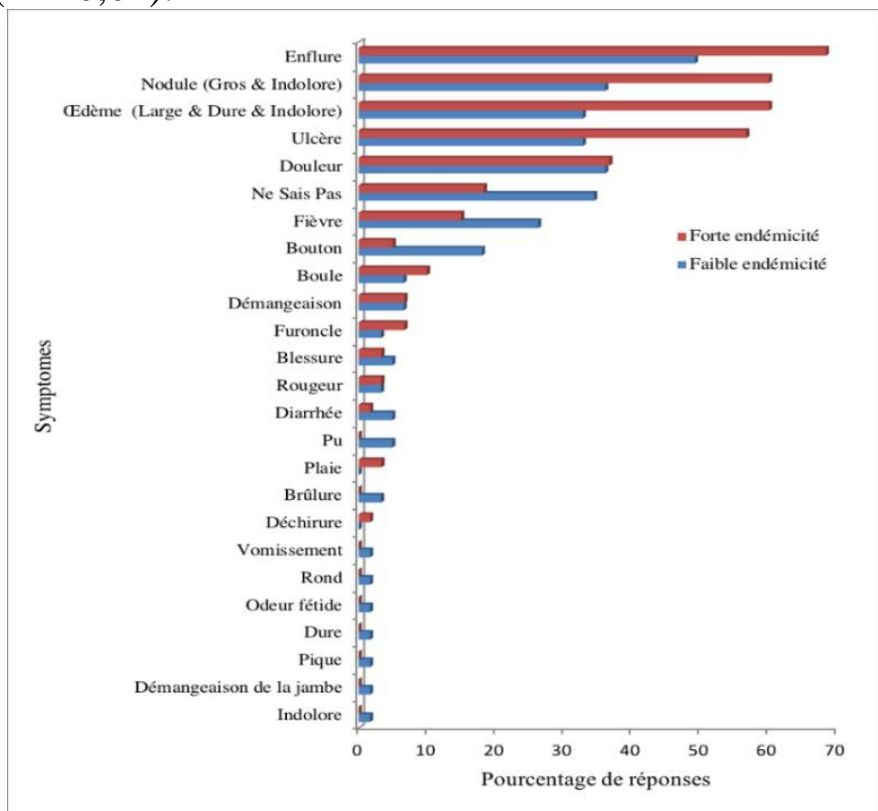

Figure 3 : Connaissance des manifestations de l'ulcère de Buruli dans les sites de forte et de faible endémicité d'ulcère de Buruli

Les fréquences des personnes pour lesquelles les plantes sont responsables de la maladie, entre les zones de forte endémicité et celles de faible endémicité, sont statistiquement différentes. Le statut du village semble être un paramètre qui influence la réponse des populations $(P=0,007)$. Les personnes interrogées qui attribuent aux plantes un rôle dans la transmission de la maladie représentent $21,76 \%$ de la population dans les sites de forte endémicité. Dans les villages de faible endémicité, cette proportion est plus 
importante et est de 45,90\%. Quant aux causes telles que l'alimentation, la boue, les microbes et les totems, elles ne sont pas reliées au statut du site (figure 4). Aucune différence statistique n'a été observée.

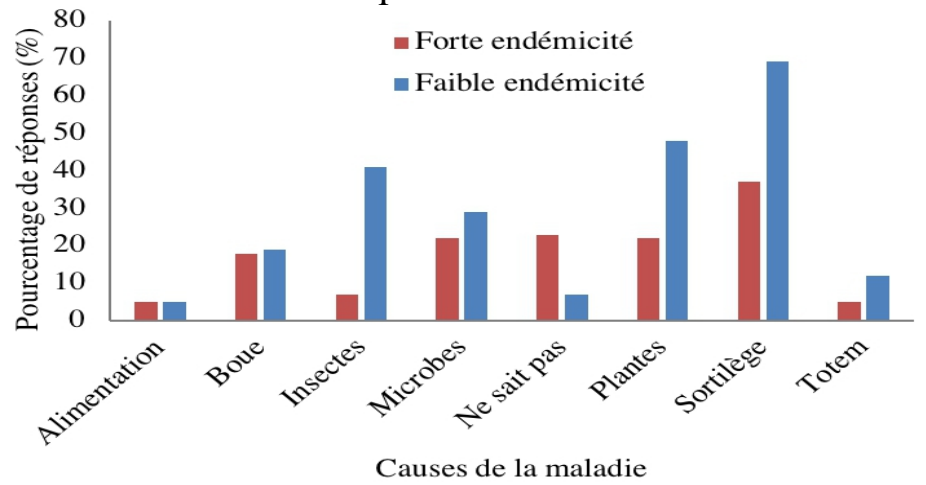

Figure 4 : Causes de l'ulcère de Buruli dans les sites de forte et de faible endémicité

En définitive, l'hypothèse relative au manque de connaissances des populations de l'ulcère de Buruli est vérifiée. En effet, elles ne reconnaissent pas toujours les symptômes de l'ulcère de Buruli. Néanmoins, elles ont contribué à l'identification d'une liste de plantes pouvant entraîner des blessures. Ces effractions cutanées peuvent être des portes d'entrée de $M$. ulcerans dans l'organisme.

\section{Discussion}

Ce travail a permis de connaître le niveau de connaissance de l'ulcère de Buruli par les populations rurales et des plantes en lien avec la transmission de la maladie. Les enquêtes se sont déroulées dans les villages de Kongodékro et Konankro (Bouaké) et dans les villages de Batéguédia et Zébra (Daloa). D'une manière générale, les résultats socio-démographiques montrent que les enfants âgés de 10 à 15 ans sont les plus affectés par la maladie. Selon Maman et al. (2018) et WHO (2016) en Afrique, plus de $50 \%$ des cas d'ulcère de Buruli enregistrés l'ont été chez des personnes âgées de moins de 17 ans. Cette forte proportion pourrait s'expliquer par le fait que les enfants sont moins attentifs aux questions d'hygiène, encore moins au port de vêtements de protection. Ils affectionnent la baignade et la pêche dans les points d'eau. Les points d'eau sont souvent très fréquentés par les enfants, qui y vont pour des motifs récréatifs (Dassi et al., 2017). Par ailleurs, leur système immunitaire encore immature les rend plus vulnérables aux maladies (Simon et al., 2015 ; Aboagye et al., 2017).

La pratique agricole est très répandue parmi les personnes interrogées. En effet, les sites visités sont des zones agricoles où les populations sont en contact avec des retenues d'eau pour l'arrosage des cultures et la riziculture dans les bas-fonds. L'activité agricole a déjà été 
indiquée comme une pratique favorisant la contamination à l'ulcère de Buruli en Côte d'Ivoire dans la région de Tiassalé (Brou et al., 2008 ; N'Krumah et al., 2016) et ailleurs en Afrique (Aboagye et al., 2017 ; Awah et al., 2018). Au Cameroun par exemple, près de 51,9 \% des personnes enquêtées par Akoachere et al. (2016) sont des agriculteurs. La pratique de l'agriculture pourrait être un facteur de risque dans l'infection à l'ulcère de Buruli, car elle augmente la probabilité de contact des populations avec les réservoirs hydrotelluriques. Ainsi, une grande partie de la population active est exposée à l'ulcère de Buruli.

Les appellations de l'ulcère de Buruli se rapportent toute à la phase d'ulcération de la maladie. Ce qui pourrait présager que les connaissances locales ne prennent pas en compte les phases initiales. Ainsi, les populations pourraient retarder de consulter les agents de santé communautaires.

Près de la moitié des personnes interrogées attribuent la transmission de l'ulcère de Buruli à une cause mystique, plus précisément à des sortilèges qui seraient lancés par des détenteurs de pouvoirs mystiques. Dans l'ensemble des sites visités, ce sont près de $52 \%$ des personnes interrogées qui ont cette position. Dans les localités de faible endémicité, l'origine mystique est reconnue par 68,85\% des enquêtés contre plus de 36,67\% dans les sites de forte endémicité. Ces résultats abondent dans le même sens que ceux de Stienstra et al. (2002) et Abel (2017) au Ghana et en Côte d'Ivoire où les populations considèrent 1'ulcère de Buruli comme le fait de sortilèges. Selon ces auteurs, dans de nombreuses communautés rurales au Ghana, la sorcellerie peut être employée pour transmettre l'ulcère de Buruli. De telles croyances sont également rapportées par Owusu \& Adamba (2012) et Anokye et al. (2018). Toutefois, il est à noter le manque d'informations sur la transmission de la maladie données aux populations. Le faible niveau de connaissance pourrait s'expliquer par le bas niveau de scolarisation dans les zones rurales, qui est estimé à $45 \%$ (World Bank, 2012). Les résultats de leur enquête révèlent que près de $38 \%$ des personnes interrogées dans le Centre de santé à Agogo au Ghana croient que l'ulcère de Buruli peut être transmis par les sorciers. C'est pour cette raison que Awah et al. (2018) recommandent la prise en compte des perceptions et des croyances traditionnelles dans l'élaboration des programmes de contrôle de l'ulcère de Buruli dans les pays africains. La possibilité que les insectes puissent transmettre $M$. ulcerans est documenté dans la littérature (Marsollier et al., 2002 ; Wallace et al., 2017). Dans ce travail, les populations dans les sites de forte endémicité (7\%), n'accordent pas autant d'importance à ce mode de transmission que dans les sites de faible endémicité (41\%); ce qui suggèrerait un manque d'informations sur les potentiels vecteurs environnementaux de l'agent responsable. Cet état de fait a pour corollaire l'absence de prise de mesures de protections adéquates telles que l'emploi de moustiquaires, de répulsifs ou de vêtements couvrant les 
membres (Kenu et al., 2014 ; Aboagye et al., 2017). Ceci augmente les risques d'exposition à la maladie. Une proportion très importante affirme ne pas connaître les causes de la maladie dans les sites de faible endémicité. Ces résultats sont analogues à ceux de Anokye et al. (2018), au Ghana. Ces derniers ont montré que près de $53 \%$ des personnes affectées ne connaissent pas les causes de l'ulcère de Buruli. Des études plus antérieures, datant de plus de 10 ans ont rapporté les mêmes faits au Ghana (Renzaho et al., 2007). L'augmentation des cas dans les pays africains peut être attribuée à ce manque de connaissances. Selon Kanga et al. (2005), la connaissance de la maladie jouerait en faveur de la réduction des cas d'ulcère de Buruli et de la limitation des séquelles liées aux complications ou au retard dans la détection de la maladie.

Dans certains cas, les populations attribuent à l'ulcère de Buruli, les symptômes que sont la diarrhée et les vomissements. De tels symptômes n'ont jamais été cités comme symptômes de la maladie (Asiedu et al., 2000). Ceci traduit donc une mauvaise connaissance de la maladie de la part des populations enquêtées, surtout dans les localités de faible endémicité. En effet, le fait qu'il n'y ait pas suffisamment de cas, contribuerait à une mauvaise connaissance de la maladie dans ces zones. Anokye et al. (2018) ont déjà rapporté au Ghana que les populations pouvaient avoir une mauvaise connaissance des signes de la maladie allant jusqu'à identifier des symptômes comme l'augmentation de l'appétit, une prise de poids et un gonflement de la peau.

La maladie est mieux connue dans les localités de forte endémicité. Ce résultat corrobore avec ceux de Renzaho et al. (2007) et Akoachere et al. (2016) qui ont rapporté un niveau très élevé de connaissance de ce problème de santé publique, dans les localités de forte endémicité. À cela s'ajoute les campagnes de sensibilisation réalisées dans les localités les plus endémiques.

Les populations affirment que la transmission de la maladie peut être liée à une ouverture de la peau, suite à une effraction par les plantes. Durant cette étude, les populations ont identifié huit espèces végétales susceptibles d'occasionner des blessures. Il s'agit de Elaeis guineensis, Imperata cylindrica, Chromolaena odorata, Combretum racemosum, Ceiba pentandra, Dioscorea spp., Saccharum officinarum et Scleria depressa. Quelques-unes de ces plantes à savoir Elaeis guineensis, Imperata cylindrica et Chromolaena odorata interviennent en médecine traditionnelle dans le traitement de l'ulcère de Buruli dans le District sanitaire de Yamoussoukro (Adjet et al., 2016). La citation de ces plantes dans les régions endémiques, pourrait expliquer le fait qu'elles constituent une inquiétude pour les populations. En outre dans cette étude, selon les populations les menaces viendraient soit des feuilles dans le cas de Elaeis guineensis et Imperata cylindrica soit des souches affleurant le sol en ce qui concerne Chromolaena odorata. Parmi ces plantes, Chromolaena 
odorata, communément appelée indépendance ou Sékou Touré par les populations, est une plante envahissante présente dans les champs en Côte d'Ivoire (Neuba et al., 2014). Elle suscite chez les populations une inquiétude car sa blessure est souvent initiatrice de plaies incurables si elles sont non aseptisées. Pour Combretum racemosum, la méfiance des populations vis-àvis de cette plante pourrait s'expliquer par la présence d'épines qu'elle porte sur sa tige. C'est le cas également de Elaeis guineensis, communément connue sous le nom de palmier à huile. Les piqûres des aiguilles de cette plante entraînent des complications qui peuvent s'apparenter quelques fois à des ulcères lorsque les plaies ne sont pas correctement traitées. Bien qu'il n'existe pas de lien direct de cause à effet entre la transmission de la maladie et ces espèces végétales, il est recommandé aux populations dans les zones endémiques de prendre des précautions lors de la manipulation de ces plantes. En effet, ces plantes pourraient porter des biofilms. Les études ont démontré que les biofilms de plantes expriment la présence de l'ADN de M. ulcerans (Tano et al., 2017).

Les appellations de l'ulcère de Buruli varient d'une région à une autre, selon la langue locale. Traduites en français, ces appellations correspondent soit à mauvaise ou vilaine plaie, grosse plaie ou encore plaie de Daloa, pour indiquer la région de la Côte d'Ivoire où l'endémie a été la plus importante dans les années 80 (Saki et al., 1995). Dans les différentes langues locales, l'appellation est en rapport avec la nature de la plaie observée. Par contre au Cameroun, l'appellation de l'ulcère de Buruli est liée à son origine mystique. Les noms «Atom», «Mbouati » ou «nbong » se traduiraient par « victime du regard d'un démon ». Elle est aussi connue sous le nom de « plaie incurable » (Grietens et al., 2012; Akoachere et al., 2016 ; Awah et al., 2018).

\section{Conclusion}

Cette étude révèle que les populations ont une connaissance fragmentaire de l'ulcère de Buruli. L'existence d'une cause mystique de la maladie demeure persistante chez les populations. Les populations ont permis d'identifier huit plantes pour lesquelles il convient d'éviter les blessures ou d'avoir recours à des mesures d'asepsie surtout dans les zones endémiques. Il convient de mener d'autres études afin d'évaluer l'affinité de M. ulcerans pour ces plantes.

\section{Remerciements}

Nous tenons surtout à remercier le Programme d'Appui Stratégique à la Recherche Scientifique (PASRES) qui a financé entièrement ce travail (Projet PASRES P-158/2015).

Les auteurs tiennent à remercier le Programme National de Lutte contre l'Ulcère du Buruli en Côte d'Ivoire (PNLUB) du ministère de la Santé 
et de l'Hygiène Publique pour les autorisations pour la réalisation de cette étude, le personnel des Districts sanitaires de Daloa et Bouaké, ainsi que les populations des villages de Batéguédia, Kongodégro, Konankro et N'Zéré qui ont permis la collecte de ces données.

\section{References:}

1. Aboagye, S. Y., Ampah, K. A., Ross, A., Asare, P., Otchere, I. D., Fyfe, J., \& Yeboah-Manu, D. (2017). Seasonal Pattern of Mycobacterium ulcerans, the Causative Agent of Buruli Ulcer, in the Environment in Ghana. Microbial Ecology, 74(2), 350-361. https://doi.org/10.1007/s00248-017-0946-6

2. Adjet, A A, Kouame, D., \& Fokou, G. (2016). Phytothérapie et lutte contre l'ulcère de Buruli dans le district sanitaire de Yamoussoukro ( Côte d'Ivoire ) : identification, description, fonction symbolique des plantes et recettes utilisées Phytotherapy against buruli ulcer in the Health Distct of Ya. Médecine et Santé Tropicales, 26, 408-413. https://doi.org/10.1684/mst.2016.0630

3. Adjet, Abel A. (2017). Itinéraires thérapeutiques des malades de l'ulcère de Buruli et difficultés de prise en charge hospitalière à Djékanou (Côte d'Ivoire). European Scientific Journal, ESJ, 13(3), 197-210. https://doi.org/10.19044/ESJ.2017.V13N3P\%P

4. Akoachere, J.-F. K. T., Nsai, F. S., \& Ndip, R. N. (2016). A Community Based Study on the Mode of Transmission, Prevention and Treatment of Buruli Ulcers in Southwest Cameroon: Knowledge, Attitude and Practices. PLOS ONE, 11(5), 1-18. https://doi.org/10.1371/journal.pone.0156463

5. Anokye, R., Acheampong, E., Mprah, K., Sarpong, E., Mprah, W. K., \& Sarpong, E. (2018). Perceived causes and risk factors of Buruli ulcer among patients at Agogo Presbyterian hospital in Ashanti Region of Ghana. BMC Research Notes, 11(1), 1-5. https://doi.org/10.1186/s13104-018-3172-5

6. Asiedu, K., Scherpbier, R., \& Raviglione, M. (2000). Buruli ulcer : Mycobacterium ulcerans infection. Geneva, Switzerland. Retrieved from

http://apps.who.int/iris/bitstream/handle/10665/66164/WHO_CDS_C PE_GBUI_2000.1.pdf?sequence=1\&isAllowed=y

7. Awah, P. K., Boock, A. U., Mou, F., Koin, J. T., Anye, E. M., Noumen, D., Nichter, M., \& Consortium, S. B. (2018). Developing a Buruli ulcer community of practice in Bankim, Cameroon: A model for Buruli ulcer outreach in Africa. PLoS Neglected Tropical Diseases, 12(3), 120. https://doi.org/10.1371/journal.pntd.0006238

8. Boni, C. C., Ehouman, E., Soro, D., Koné, M. W., Bakayoko, A., 
Dembélé, F., Bauthiré, K., \& Dosso, M. (2017). Étude comparative de la flore aux abords des cours d'eau dans les zones hypo et hyper endémiques d'ulcère de Buruli en Côte d'Ivoire. International Journal of Biological and Chemical Sciences, 11(3), 1254-1270. https://doi.org/10.4314/ijbcs.v11i3.26

9. Brou, T., Broutin, H., Elguero, E., Asse, H., \& Guegan, J.-F. (2008). Landscape Diversity Related to Buruli Ulcer Disease in Côte d'Ivoire. PLoS Neglected Tropical Diseases, 2(7), 1-9. https://doi.org/10.1371/journal.pntd.0000271

10. Cooper, J. D., Costello, A. G., \& Shaw, P. H. (2017). A Comparison of Extremity Thrombosis Rates in Adolescent and Young Adult Versus Younger Pediatric Oncology Patients at a Children's Hospital. Journal of Adolescent and Young Adult Oncology, 6(1), 62-66. https://doi.org/10.1089/jayao.2016.0044

11. Dassi, C., Mosi, L., Narh, C., Quaye, C., Konan, D., Djaman, J., \& Bonfoh, B. (2017). Distribution and Risk of Mycolactone-Producing Mycobacteria Transmission within Buruli Ulcer Endemic Communities in Côte d'Ivoire. Tropical Medicine and Infectious Disease, 2(1), 1-3. https://doi.org/10.3390/tropicalmed2010003

12. De Ketele, J.-M., \& Roegiers, X. (2015). Méthodologie du recueil d'informations: fondements des méthodes d'observation, de questionnaire, d'interview et d'étude de documents (5e édition). Louvain-la-Neuve, Belgique: De Boeck Supérieur.

13. De Zeeuw, J., Alferink, M., Barogui, Y. T., Sopoh, G., Phillips, R. O., van der Werf, T. S., Loth, S., Molenbuur, B., Plantinga, M., Ranchor, A. V., \& Stienstra, Y. (2015). Assessment and Treatment of Pain during Treatment of Buruli Ulcer. PLoS Neglected Tropical Diseases, 9(9), 1-10. https://doi.org/10.1371/journal.pntd.0004076

14. Garapati, P., Pal, B., Siddiqui, N. A., Bimal, S., Das, P., Murti, K., \& Pandey, K. (2018). Knowledge, stigma, health seeking behaviour and its determinants among patients with post kalaazar dermal leishmaniasis, Bihar, India. PLoS ONE, 13(9), 1-13. https://doi.org/10.1371/journal.pone.0203407

15. Garchitorena, A., Ngonghala, C. N., Guegan, J.-F., Texier, G., Bellanger, M., Bonds, M., \& Roche, B. (2015). Economic inequality caused by feedbacks between poverty and the dynamics of a rare tropical disease: the case of Buruli ulcer in sub-Saharan Africa. Proceedings. Biological Sciences, 282(1818), 1-9. https://doi.org/10.1098/rspb.2015.1426

16. Giles-Vernick, T., Owona-Ntsama, J., Landier, J., \& Eyangoh, S. (2015). The puzzle of Buruli ulcer transmission ethno-ecological history and the end of "love" in the Akonolinga District, Cameroon. 
Social Science \& Medicine, 129, 2027.https://doi.org/https://doi.org/10.1016/j.socscimed.2014.03.008

17. Grietens, P. K., Toomer, E., Um Boock, A., Hausmann-Muela, S., Peeters, H., Kanobana, K., Gryseels, C., \& Ribera, J. M. (2012). What Role Do Traditional Beliefs Play in Treatment Seeking and Delay for Buruli Ulcer Disease?-Insights from a Mixed Methods Study in Cameroon. $\quad P L O S \quad O N E, \quad 7(5), \quad 1-8$. https://doi.org/10.1371/journal.pone.0036954

18. Handicap. (2013). Enquête CAP sur l'ulcère de Buruli au sein de la population de Zio et Yoto. Document interne, Handicap International, Lomé, Togo.

19. Hotez, P. J., \& Kamath, A. (2009). Neglected Tropical Diseases in Sub-Saharan Africa: Review of Their Prevalence, Distribution, and Disease Burden. PLoS Neglected Tropical Diseases, 3(8), 1-10. https://doi.org/10.1371/journal.pntd.0000412

20. INS. (2015a). Répertoire des localités (2014): Région du Gbèkè. Abidjan, Côte d'Ivoire: INS. Retrieved from www.ins.ci/n/documents/rgph/GBEKE.pdf

21. INS. (2015b). Répertoire des localités (2014): Région du HautSassandra. Abidjan, Côte d'Ivoire: INS. Retrieved from http://www.ins.ci/n/documents/rgph/HAUT_SASSANDRA.pdf

22. Kanga, J. M., Kacou, E. D., Kouamé, K., Kassi, K., Kaloga, M., Yao, J. K., Dion-Lainé, M., Avoaka, L. E., Yoboué-Yao, P., Sangaré, A., Ecra, J. E., Ahogo, C., Djédjé, M. S., Kadiri, A. J., \& Ayé, C. (2005). La lutte contre l'ulcère de Buruli. Expérience de la Côte d'Ivoire. Bulletin de La Société de Pathologie Exotique, 99, 34-38.

23. Kenu, E., Nyarko, K. M., Seefeld, L., Ganu, V., Käser, M., Lartey, M., Calys-Tagoe, B. N. L., Koram, K., Adanu, R., Razum, O., Afari, E., \& Binka, F. N. (2014). Risk Factors for Buruli Ulcer in Ghana-A Case Control Study in the Suhum-Kraboa-Coaltar and Akuapem South Districts of the Eastern Region. PLoS Neglected Tropical Diseases, 8(11), 1-9. https://doi.org/10.1371/journal.pntd.0003279

24. Khanna, R., Meena, R. N., Mukunda, M. V., \& Khanna, S. (2016). Infective Leg Ulcers. In A. K. Khanna \& T. S. K. (Eds.), Ulcers of the Lower Extremity (Springer, pp. 267-274). Varanasi: Banaras Hindu University. https://doi.org/10.1007/978-81-322-2635-2

25. Koka, E., Yeboah-Manu, D., Okyere, D., Adongo, P. B., \& Ahorlu, C. K. (2016). Cultural Understanding of Wounds, Buruli Ulcers and Their Management at the Obom Sub-district of the Ga South Municipality of the Greater Accra Region of Ghana. PLoS Neglected Tropical Diseases, 10(7), 1-20. https://doi.org/10.1371/journal.pntd.0004825

26. Maman, I., Tchacondo, T., Kere, A. B., Piten, E., Beissner, M., 
Kobara, Y., Kossi, K., Badziklou, K., Wiedemann, F. X., Amekuse, K., Bretzel, G., \& Karou, D. S. (2018). Risk factors for Mycobacterium ulcerans infection (Buruli Ulcer) in Togo - a casecontrol study in Zio and Yoto districts of the maritime region. BMC Infectious Diseases, 18(48), 1-11. https://doi.org/10.1186/s12879018-2958-3

27. Marsollier, L., Robert, R., Aubry, J., André, J.-P. Saint, Kouakou, H., Legras, P., Manceau, A.-L., Chetaou, M., \& Carbonnelle, B. (2002). Aquatic Insects as a Vector for Mycobacterium ulcerans. Applied and Environmental Microbiology, 68(9), 4623-4628. https://doi.org/10.1128/AEM.68.9.4623-4628.2002

28. Marston, B. J., Diallo, M. O., Horsburgh, C. R. J., Diomande, I., Saki, M. Z., Kanga, J.-M., Patrice, G., Lipman, H. B., Ostroff, S. M., \& Good, R. C. (1995). Emergence of Buruli Ulcer Disease in the Daloa Region of Côte D'Ivoire. The American Journal of Tropical Medicine and Hygiene, 52(3), 219-224. https://doi.org/10.4269/ajtmh.1995.52.219

29. McIntosh, M., Williamson, H., Benbow, M. E., Kimbirauskas, R., Quaye, C., Boakye, D., Small, P., \& Merritt, R. (2014). Associations Between Mycobacterium ulcerans and Aquatic Plant Communities of West Africa: Implications for Buruli Ulcer Disease. EcoHealth, 11(2), 184-196. https://doi.org/10.1007/s10393-013-0898-3

30. Merritt, R. W., Walker, E. D., Small, P. L. C., Wallace, J. R., Johnson, P. D. R., Benbow, M. E., \& Boakye, D. A. (2010). Ecology and Transmission of Buruli Ulcer Disease: A Systematic Review. PLoS Neglected Tropical Diseases, 4(12), 1-15. https://doi.org/10.1371/journal.pntd.0000911

31. N'Krumah, R. T. A. S., Koné, B., Tiembre, I., Cissé, G., Pluschke, G., Tanner, M., \& Utzinger, J. (2016). Socio-Environmental Factors Associated with the Risk of Contracting Buruli Ulcer in Tiassalé, South Côte d'Ivoire: A Case-Control Study. PLoS Neglected Tropical Diseases, 10(1), 1-15. https://doi.org/10.1371/journal.pntd.0004327

32. Neuba, D. F. R., Malan, D. F., Koné, M., \& Kouadio, Y. L. (2014). Inventaire préliminaire des plantes envahissantes de la Côte d'Ivoire. Journal of Animal and Plant Sciences (JAPS), 22(2), 3439-3445.

33. O’Brien, D. P., Jeanne, I., Blasdell, K., Avumegah, M., \& Athan, E. (2019). The changing epidemiology worldwide of Mycobacterium ulcerans . Epidemiology and Infection, 147(5), 1-8. https://doi.org/10.1017/S0950268818002662

34. Owusu, A. Y., \& Adamba, C. (2012). Household Perceptions, Treatment-Seeking Behaviors and Health Outcomes for Buruli Ulcer Disease in a Peri-Urban District in Ghana. Advances in Applied 
Sociology, 02(03), 179-186. https://doi.org/10.4236/aasoci.2012.23024

35. Pearson, G. (2018). Understanding perceptions on "Buruli" in northwestern Uganda: A biosocial investigation. PLoS Neglected Tropical Diseases, 12(7), 1-18. https://doi.org/10.1371/journal.pntd.0006689

36. PNLUB. (2013). Bilan d'activités 2013 du Programme National de Lutte contre l'ulcère de Buruli. Document interne, Programme Natinal de Luttre contre l'Ulcère de Buruli (PNLUB), Abidjan, Côte d'Ivoire.

37. PNLUB. (2014). Bilan d'activités 2014 du Programme National de Lutte contre l'Ulcère de Buruli. Document interne, Programme National de Lutte contre l'Ulcère de Buruli (PNLUB), Abidjan, Côte d'Ivoire.

38. Portaels, F., Meyers, W. M., Ablordey, A., Castro, A. G., Chemlal, K., de Rijk, P., Elsen, P., Fissette, K., Fraga, A. G., Lee, R., Mahrous, E., Small, P. L. C., Stragier, P., Torrado, E., Van Aerde, A., Silva, M. T., \& Pedrosa, J. (2008). First cultivation and characterization of Mycobacterium ulcerans from the environment. PLoS Neglected Tropical Diseases, 2(3). https://doi.org/10.1371/journal.pntd.0000178

39. Renzaho, A. M. N., Woods, P. V., Ackumey, M. M., Harvey, S. K., \& Kotin, J. (2007). Community-based study on knowledge, attitude and practice on the mode of transmission, prevention and treatment of the Buruli ulcer in Ga West District, Ghana. Tropical Medicine \& International Health, 12(3), 445-458. https://doi.org/10.1111/j.13653156.2006.01795.x

40. Röltgen, K., \& Pluschke, G. (2015). Mycobacterium ulcerans Disease (Buruli Ulcer): Potential Reservoirs and Vectors. Current Clinical Microbiology Reports, 2(1), 35-43. https://doi.org/10.1007/s40588015-0013-3

41. Ross, B. C., Johnson, P. D., Oppedisano, F., Marino, L., Sievers, A., Stinear, T., Hayman, J. A., Veitch, M. G., \& Robins-Browne, R. M. (1997). Detection of Mycobacterium ulcerans in environmental samples during an outbreak of ulcerative disease. Applied and Environmental Microbiology, 63(10), 4135-4138.

42. Saki, M. Z., Diomandé, I., Diallo, M. O., Marston, B. J., Horsburgh, C. R., Ostroff, S. M., Good, R. C., Lipman, H. B., Patrice, G., \& Kanga, J.-M. (1995). Emergence of Buruli Ulcer Disease in the Daloa Region of Côte d'Ivoire. The American Journal of Tropical Medicine and Hygiene, 52(3), 219-224. https://doi.org/10.4269/ajtmh.1995.52.219

43. Simon, A. K., Hollander, G. A., Mcmichael, A., \& Mcmichael, A. (2015). Evolution of the immune system in humans from infancy to old age. Proc. Biol. Sci., 282(1821), 1-12. 
https://doi.org/10.1098/rspb.2014.3085

44. Sopoh, G., \& Asiedu, K. (2016). Buruli Ulcer in Sub-Saharan Africa. In J. Gyapong \& B. Boatin (Eds.), Neglected Tropical Diseases-SubSaharan Africa (pp. 15-43). Basel, Switzerland: Springer International Publishing. https://doi.org/10.1007/978-3-319-25471-5_2

45. Standley Id, C., Boyce, M. R., Klineberg, A., Essix, G., \& Katz, R. (2018). Organization of oversight for integrated control of neglected tropical diseases within Ministries of Health. PLoS Neglected Tropical Diseases, 12(11), 1-14. https://doi.org/10.1371/journal.pntd.0006929

46. Stienstra, Y., van der Graaf, W. T. A., Asamoa, K., \& van der Werf, T. S. (2002). Beliefs and attitudes toward Buruli ulcer in Ghana. The American Journal of Tropical Medicine and Hygiene, 67(2), 207-213.

47. Tai, A. Y. C., Athan, E., Friedman, N. D., Hughes, A., Walton, A., \& O'Brien, D. P. (2018). Increased Severity and Spread of Mycobacterium ulcerans , Southeastern Australia. Emerging Infectious Diseases, 24(1), 58-64. https://doi.org/10.3201/eid2401.171070

48. Tano, M. B., Dassi, C., Mosi, L., Koussémon, M., \& Bonfoh, B. (2017). Molecular characterization of mycolactone producing mycobacteria from aquatic environments in Buruli ulcer non-endemic areas in Côte d'Ivoire. International Journal of Environmental Research and Public Health, 14(2), 1-10. https://doi.org/10.3390/ijerph14020178

49. Tschakert, P., Ricciardi, V., Smithwick, E., Machado, M., Ferring, D., Hausermann, H., \& Bug, L. (2016). Situated knowledge of pathogenic landscapes in Ghana: Understanding the emergence of Buruli ulcer through qualitative analysis. Social Science and Medicine, 150(January 2016), 160-171. https://doi.org/10.1016/j.socscimed.2015.12.005

50. Wallace, J. R., Mangas, K. M., Porter, J. L., Marcsisin, R., Pidot, S. J., Howden, B., Omansen, T. F., Zeng, W., Axford, J. K., Johnson, P. D. R., \& Stinear, T. P. (2017). Mycobacterium ulcerans low infectious dose and mechanical transmission support insect bites and puncturing injuries in the spread of Buruli ulcer. PLoS Neglected Tropical Diseases, 11(4), 1-16. https://doi.org/10.1371/journal.pntd.0005553

51. WHO. (2016). WHO | The history of GBUI. Retrieved September 27, 2018, from http://www.who.int/buruli/gbui/en/

52. WHO. (2017). Global Health Observatory data repository : Number of new reported cases Data by country. Retrieved September 27, 2018, from http://apps.who.int/gho/data/node.main.A1631

53. Williamson, H. R., Mosi, L., Donnell, R., Aqqad, M., Merritt, R. W., \& Small, P. L. C. (2014). Mycobacterium ulcerans Fails to Infect 
through Skin Abrasions in a Guinea Pig Infection Model: Implications for Transmission. PLoS Neglected Tropical Diseases, 8(4), 1-10. https://doi.org/10.1371/journal.pntd.0002770

54. World Bank. (2012). Côte d'Ivoire - Emergency Basic Education Support Project. Document interne, The World Bank, Whashington DC, USA.

55. Yusra, H. K., Azmi, S., Amer, H. K., \& Tauqeer, H. M. (2014). Knowledge, attitude and practice (KAP) survey of osteoporosis among students of a tertiary institution in Malaysia. Tropical Journal of Pharmaceutical Research, 13(1), 155-162. Retrieved from https://www.ajol.info/index.php/tjpr/article/view/101073

56. Zogo, B., Djenontin, A., Carolan, K., Babonneau, J., Guegan, J.-F., Eyangoh, S., \& Marion, E. (2015). A Field Study in Benin to Investigate the Role of Mosquitoes and Other Flying Insects in the Ecology of Mycobacterium ulcerans. PLOS Neglected Tropical Diseases, 9(7), 1-12. https://doi.org/10.1371/journal.pntd.0003941 